

\section{Sumário}

Ordenamiento ambiental del terRitorio y participación CiUdadana en Argentina: aportes posibles DESDE EL DERECHO 2

María Valeria Berros

Matéria ambiental nos tribunais da Austrália e nova Zelândia ................................................... 17 Marcio Oliveira Portella

Conservação da BIODIVERSIDADE E POLÍtICAS PÚbLICAS PARA AS ÁREAS PROTEGIDAS NO BRASIL: DESAFIOS E

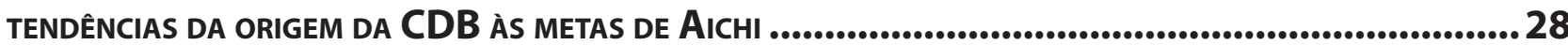

Ana Paula Leite Prates e Marta de Azevedo Irving

Dimensões linguísticas dA DESIGUALDADE No BrASIL: OS DIVERSOS NOMES LEGAIS DE UM MESMO FENÔMENo

Jefferson Carús Guedes

ECONOMIA, ÉTICA E TRIBUTAÇÃO: DOS FUNDAMENTOS DA DESIGUALDADE

Rafael Köche e Marciano Buffon

A REPARTIÇÃO de RENDAS ENTRE OS ENTES DA FEDERAÇÃO E SUA REPERCUSSÃo NA CARACTERIZAÇÃo DO FEDERALISMO BRASILEIRO: UMA INVESTIGAÇÃO ACERCA DO DESENVOLVIMENTO DO FEDERALISMO FISCAL-FINANCEIRO NO BRASIL.

Raquel Mousinho de Moura Fé

UMA AVALIAÇÃO DO ÍNDICE DE INCLUSÃO FINANCEIRA NOS ESTADOS DO NORDESTE BRASILEIRO

Diego Araujo Reis e Osvaldo Sousa Ventura

Fatores determinantes do Índice de Desenvolvimento Humano dos Municípios do Estado de São

PaUlo

Leandro Campi Prearo, Maria Clara Maraccini e Maria do Carmo Romeiro

As políticas públicas de acesso e difusão da cultura no Brasil e o caso do Programa Nacional de Apoio à Cultura. 157

Telma Rocha Lisowski

MoldANDO A "RESERVA do POSSível" NO TEMPO: A SUSTENTABILIDADE FISCAL COMO DIREITO DIFUSO FUNDAMENTAL. 171

Leonardo Romero Marino 
O PODER JUDICIÁRIO E A EFETIVAÇÃO DO DIREITO À SAÚDE

João Luis Nogueira Matias e Águeda Muniz

EDUCAÇÃo SUPERIOR NO BRASIL: OFERTA NA OMC, UMA REFLEXÃO DESMISTIFICADA, COM BASE EM DADOS ESTATÍSTICOS 208

Marcel Vitor Guerra

A GARANTIA DO DIREITO À EDUCAÇÃO DE CRIANÇAS E ADOLESCENTES NO CONTEXTO DAS POLÍTICAS PÚBLICAS BRASILEIRAS

André Viana Custódio e Rafael Bueno da Rosa Moreira

A POLÍCIA JUDICIÁRIA E O COMBATE À CRIMINALIDADE

Júlio Lopes Hott

Processo eleitoral e políticas Públicas: influÊnCias ReCíProcas.

Pablo Malheiros da Cunha Frota

SOCIEDADES PRIMITIVAS E DIREITO CONTEMPORÂNEO: DE QUE FORMA A JUSTIÇA TRIBAL PODE NOS AJUDAR A REPENSAR A NOSSA JUSTIÇA 303

Amanda Rodrigues e Tiago Themudo

DIREITO PRIVADO, JUSTIÇA DISTRIBUTIVA E O ARGUMENTO DA DUPLA DISTORÇÃO: UMA REVISÃO DA LITERATURA ...........318 Leandro Martins Zanitelli

DistorçõES NO CONTROLE CADASTRAL DAS ENTIDADES SINDICAIS E CONTRIBUIÇÃO SINDICAL Ricardo Bravo

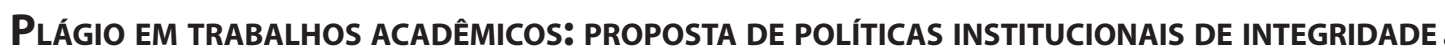
354 Maria Edelvacy Pinto Marinho e Marcelo Dias Varella 


\title{
Uma avaliação do índice de inclusão financeira nos estados do nordeste brasileiro*
}

\author{
An assessment of the financial inclusion \\ index in the states of northeastern Brazil
}

\author{
Diego Araujo Reis** \\ Osvaldo Sousa Ventura***
}

\section{Resumo}

O presente trabalho visa analisar o grau de inclusão financeira dos estados do nordeste brasileiro, em 2000, 2005 e 2010, a partir do índice de inclusão financeira (IIF) calculado pelo Banco Central do Brasil (BCB), no relatório de inclusão financeira (RIF II). Dos dados apresentados, verificou-se que todos os estado experimentaram incrementos no grau de inclusão financeira para o momento em exame. Também foi realizada análise de correlação entre o IIF e indicadores de desenvolvimento da FIRJAN em suas três dimensões (Emprego \& Renda, Educação e Saúde), utiliza-se proxy de indicadores de bem-estar. $\mathrm{O}$ resultado encontrado para esse último ponto revelou que há forte correlação entre esses índices, levantando a hipótese de que a inclusão financeira experimentada pelos estados nordestinos pode ter contribuído para desenvolvimento econômico da região e, consequentemente, de sua população.

Palavras-chave: Inclusão financeira. Banco Central do Brasil. Nordeste.

* Recebido em 14/10/2014 Aprovado em 30/01/2015

** Mestre em Desenvolvimento Regional e Gestão de Empreendimentos Locais pelo Núcleo de Pós-graduação em Economia da Universidade Federal de Sergipe (NUPEC/UFS). Professor (substituto) de Contabilidade Social pelo Departamento de Economia da UFS. Consultor de Inovação e Técnico de Pesquisa. Email: diegoaraujoreis@hotmail.com

*** Mestre em Desenvolvimento Regional e Gestão de Empreendimentos Locais pelo Núcleo de Pós-graduação em Economia da Universidade Federal de Sergipe (NUPEC/UFS). Economista da Empresa Brasileira de Correios e Telégrafos (ECT) em Sergipe. E-mail: osvaldo.ventura@uol.com.br

\section{Abstract}

This study aims to analyze the degree of financial inclusion of the states in northeastern Brazil, in 2000, 2005 and 2010, the index for financial inclusion (IIF) calculated by the Central Bank of Brazil (BCB), the financial inclusion report (RIF II). From the data presented, it was verified that all state experienced increases in the degree of financial inclusion for the moment under consideration. From the correlation analysis between the IIF and development indicators FIRJAN in its three dimensions (Employment \& Income, Education and Health), used here as a proxy indicator of well-being. The results found for the latter point was that there is a strong correlation between these indices, raising the hypothesis that financial inclusion experienced by the northeastern states may have contributed to the economic development of the region and therefore of its population.

Keywords: Financial inclusion. Central Bank of Brazil. Northeast. 


\section{INTRODUÇÃO}

Nos últimos anos, o Banco Central do Brasil (BCB) vem desenvolvendo uma série de políticas de inclusão financeira, com o intuito de promover o acesso ao Sistema Financeiro Nacional para milhares de brasileiros. Essas políticas estão alicerçadas em pesquisas acadêmicas, estudos elaborados pela própria instituição e por outros organismos. Para melhor compreender o tema, o BCB desenvolveu em 2011 um Índice de Inclusão Financeira (IIF) para o Brasil, como forma de realizar o acompanhamento da evolução do processo de inclusão financeira nas unidades da federação. O IIF, assim como outros dados e informações, servem para consubstanciar a tomada de decisão no que tange à implementação de políticas inclusivas nessa seara.

A concepção de que a inclusão financeira é catalisador do desenvolvimento sustenta e movimenta as ações do BCB. Essa instituição é o principal agente de articulação, coordenação e organização das ações de estímulo para inclusão financeira da população. Suas ações vêm envolvendo tanto o setor público como o setor privado na busca de alternativas para inclusão financeira, como por exemplo, os mecanismos para a bancarização da população, o modelo de correspondentes e o aperfeiçoamento do marco regulatório do cooperativismo de crédito. Esses mecanismos, dentre outros, buscam oferecer serviços financeiros à parcela da população que se encontra excluída do Sistema Financeiro Nacional.

Mesmo com os esforços empreendidos nos últimos anos, há ainda 16 milhões de brasileiros que não têm conta bancária e não usam o sistema financeiro para o pagamento de contas, conforme Figueiredo ${ }^{1}$. Ao mesmo tempo, esses potenciais usuários provavelmente necessitarão de educação financeira para usar corretamente as ferramentas bancárias disponíveis. Por essa razão, justifica-se a interferência do BCB, em desenvolver uma política efetiva de inclusão financeira no Brasil. Entretanto, a criação de políticas que busquem incluir a população precisa estar embasada em estudos e pesquisas, como forma de reduzir erros e elevar a eficiência sistêmica.

Assim, o adequado conhecimento e entendimento dos problemas que limitam o processo de inclusão financeira é passo fundamental para o desenvolvimento de políticas públicas que visam mitigar os problemas apresentados nessa área. Nesse sentido, os Relatórios de Inclusão Financeira (RIF), desenvolvidos pelo BCB em 2010 e 2011, visam suprir parte dessa lacuna, uma vez que seu conteúdo é composto por informações relevantes sobre o tema no país, tais como parâmetros, indicadores e resultados que podem ser comparados e avaliados.

A investigação realizada no presente artigo, por sua vez, estabelece atenção especial aos resultados do Índice de Inclusão Financeira (IIF) construído e disponibilizado pelo BCB no RIF de 2011. O referido índice é composto a partir de indicadores agregados em três dimensões, a saber: acesso — disponibilidade geográfica, acesso - disponibilidade demográfica e Uso. E ele permite o acompanhamento da evolução da inclusão financeira nos últimos anos em termos de acesso e uso de serviços financeiros em diferentes unidades geográficas do país.

Pelo fato de nos últimos anos os estados do nordeste brasileiros virem apresentando taxas de crescimento econômico superiores às outras unidades da federação, delimitou-se o escopo do presente trabalho à análise da inclusão financeira nessa região. Portanto, o objetivo desta investigação consiste em analisar a inclusão financeira dos estados nordestinos a partir do Índice de Inclusão Financeira (IIF), disponível para os anos de 2000, 2005 e 2010. Outra finalidade deste trabalho é examinar a correlação entre o resultado do IIF e o Índice Firjan de Desenvolvimento Municipal (IFDM), para assim se comparar desenvolvimento financeiro com o desenvolvimento econômico da região.

O trabalho está organizado em 4 sessões, além desta breve introdução. A sessão 2 apresenta sucintamente a revisão da literatura sobre as relações entre desenvolvimento financeiro e o crescimento econômico. Expõe-se o debate das experiências de mensuração da inclusão financeira no mundo e no Brasil, bem como uma análise do processo de inclusão financeira no Brasil, com destaque para os correspondentes, as cooperativas de crédito

1 FIGUEIREDO, Odail. Inclusão financeira: conquistas. Revista Rumos, Brasília, n. 52, p. 52-55, maio/jun. 2012. 
e o papel das microfinanças nesse processo. A sessão 3 trata da metodologia do presente estudo. Na sessão 4, expõem-se e analisam-se os resultados do IIF para os estados nordestinos, assim como é avaliada as correlações entre o IIF e IFDM. Por fim, na sessão 5 apresentam-se as considerações finais acerca do presente estudo.

\section{Debate SObre o tema}

\subsection{Desenvolvimento financeiro e crescimento econômico}

Para o $\mathrm{BCB}^{2}$, a inclusão financeira é vista como propulsora do desenvolvimento econômico. Este argumento está respaldado nos estudos de Levine ${ }^{3}$, que ao realizar testes de causalidade, encontrou evidências estatísticas de que o desenvolvimento financeiro estimula o crescimento econômico. A ideia é que a incorporação de mais indivíduos usufruindo ativamente do sistema financeiro estimulará, grosso modo, o crescimento econômico.

Matos $^{4}$ observa que a hipótese de que o desenvolvimento financeiro estimula o crescimento econômico tem encontrado suporte a partir evidências empíricas, extraídas, em geral, de dados em cross-section de países, nos estudos de Levine ${ }^{5}$, Darrat ${ }^{6}$, Khan e Senhadji ${ }^{7}$, Lee $^{8}$ e Lee'.

Schumpeter ${ }^{10}$, no início do século XX, já reconhecia a complementariedade entre o sistema financeiro e a atividade econômica. Ele observou que os serviços financeiros são essenciais, pois permite aos usuários um acesso mais amplo ao crédito, expandindo, assim, as oportunidades econômicas, além de auxiliar a inovação tecnológica e o desenvolvimento econômico.

No entanto, há na literatura inúmeras controvérsias no que diz respeito à hipótese de que o desenvolvimento do sistema financeiro contribui para estimular o crescimento econômico. De acordo com Matos ${ }^{11}$, são identificadas na literatura três visões, as quais oscilam entre as hipóteses de determinação conjunta, de Greenwood e Jovanovic ${ }^{12}$; a de negação da existência, de Modigliani e Miller ${ }^{13}$; e a defesa da relação inversa de que o crescimento econômico estimula o desenvolvimento do sistema financeiro, de Arestis e Demetria-

2 BANCO CENTRAL DO BRASIL, BCB. Relatório de inclusão financeira. Brasília: BCB, 2011.

3 LEVINE, R. Financial development and economic growth: views and agenda. Journal of Economic Literature, Nashville, n. 35, p. 688-726, quarterly, 1997.

4 MATOS, O. C. Inter-relações entre desenvolvimento financeiro, exportações e crescimento econômico: análise da experiência brasileira. Notas Técnicas do Banco Central do Brasil, Brasília, n. 40, out. 2003.

5 LEVINE, R. Financial development and economic growth: views and agenda. Journal of Economic Literature, Nashville, n. 35, p. 688-726, quarterly, 1997.

6 DARRAT, A. F. Are financial deepening and economic growth causality related?: another look at the evidence. International Economic Journal, Seoul, v. 13, n. 3, p. 19-35, autumn 1999.

7 KHAN, M. S.; SENHADJI, A. S. Threshold effects in the relationship between inflation and growth. IMF Working Paper WP/00/110, Washington, June 2000.

8 LEE, J. Financial development by learning. Journal of Development Economics, Republic of Korea, n. 50, p. 147-64, quarterly, 1996.

9 LEE, B. Financial development and economic growth: the role of information accumulation. Economic Papers, Australia, v. 4, n. 1, p. 1-39, quarterly, 2001.

10 SCHUMPETER, J. A. A teoria do desenvolvimento econômico. São Paulo: Abril Cultural, 1982.

11 MATOS, O. C. Inter-relações entre desenvolvimento financeiro, exportações e crescimento econômico: análise da experiência brasileira. Notas Técnicas do Banco Central do Brasil, Brasília, n. 40, out. 2003.

12 GREENWOOD, J.; JOVANOVIC, B. Financial development, growth, and the distribution of income. Journal of Political Economy, Chicago, v. 98, n. 5, part 1, p. 1076-1107, quarterly, 1990.

13 MODIGLIANI, F.; MILLER, M. H. The cost of capital, corporation finance and the theory of investment. American Economic Review, Pittsburgh, n. 48, p. 261-97, monthly, 1958. 
$\operatorname{des}^{14}$, e Demetriades e Hussein ${ }^{15}$

O argumento, que se pode ler, por exemplo, na exposição dos Relatórios de Inclusão Financeira (RIF), é que a adequada expansão da inclusão financeira viabiliza o acesso à economia formal, colaborando para maior crescimento econômico, que, por sua vez, promove o acesso de mais indivíduos à economia e ao Sistema Financeiro Nacional, mobilizando poupança e investimento para o crescimento do setor produtivo ${ }^{16}$.

Intuitivamente a elucidação supramencionada é logicamente válida. A inclusão financeira, em especial o acesso e uso a serviços tais como, crédito, produtos de poupança, pagamentos e de transferências em geral, permitem fortalecer os atores sociais contra eventuais choques econômicos particulares, uma vez que sua capacidade de ampliar ou estabilizar renda pode ser potencializado pelos serviços financeiros.

\subsection{Experiências de mensuração da inclusão financeira no mundo}

No cenário internacional, a inclusão financeira é assunto de relevância, uma vez que há o reconhecimento de sua importância como instrumento de desenvolvimento econômico e social. Segundo o BCB ${ }^{17}$, em 2009 ocorreu o encontro de Cúpula do G20, em que os líderes de estado comprometeram-se a elevar o nível de inclusão financeira no mundo, já que as livres forças de mercado não são capazes de resolver o problema da exclusão financeira.

A Cúpula do G20 criou o Financial Inclusion Experts Group (FIEG). Em seus trabalhos, o FIEG diagnosticou que $90 \%$ da população financeiramente excluída residem em países em desenvolvimento e de menor renda, o que demanda maior fortalecimento do papel dos Estados nacionais de países em desenvolvimento no processo de inclusão financeira global ${ }^{18}$.

As interferências do Estado no mercado financeiro devem ser precedidas de estudos que permitam a mensuração para o mapeamento e acompanhamento do processo de inclusão financeira. Isso para que sejam identificadas as regiões e grupos prioritários das políticas de inclusão financeira.

A mensuração da inclusão financeira é imprescindível para os países que visam superar o atual estágio de exclusão financeira. A atividade de mensurar visa verificar o grau de inclusão de todos os membros de uma economia (famílias, empresas, etc.) aos serviços financeiros, em especial os serviços bancários: abertura de conta corrente e poupança, realização de pagamentos e de recebimentos, a tomada de empréstimos (acesso ao crédito), dentre outros importantes serviços.

A mensuração do nível de inclusão financeira, porém, é relativamente nova e, na sequência, busca-se apresentar o que a literatura vem tratando sobre o assunto. Nesse quesito convém destacar a contribuição de Sarma ${ }^{19}$, que propôs um Índice de Inclusão Financeira multidimensional desenvolvido de acordo com índices de desenvolvimento humano, a exemplo do IDH. Esse índice capta informações sobre várias dimensões da inclusão financeira (penetração, disponibilidade e uso) e é um índice com um único dígito, situado entre 0 e 1, em que 0 indica a exclusão financeira completa e 1 indica a absoluta inclusão financeira.

14 ARESTIS, P.; DEMETRIADES, P. Finance and growth: is Schumpeter right? Análise Econômica, Porto Alegre, v. 6, n. 30, p. 5-21, semestral, 1998.

15 DEMETRIADES, P. O.; HUSSEIN, K. A. Does financial development cause economic growth?: time series evidence from 16 countries. Journal of Development Economics, Republic of Korea, n. 51, p. 387-411, quarterly, 1996.

16 BANCO CENTRAL DO BRASIL (BCB). Relatório de inclusão financeira. Brasília: BCB, 2011.

17 BANCO CENTRAL DO BRASIL (BCB). Relatório de inclusão financeira. 2010. Disponível em: <http://www.bcb.gov.br/Nor/ relincfin/relatorio_inclusao_financeira.pdf>. Acesso em: 06 jul. 2012.

18 BANCO CENTRAL DO BRASIL (BCB). Relatório de inclusão financeira. 2010. Disponível em: <http://www.bcb.gov.br/Nor/ relincfin/relatorio_inclusao_financeira.pdf $>$. Acesso em: 06 jul. 2012.

19 SARMA, M. Index of financial inclusion: a measure of financial sector inclusiveness. Working Paper, India, n. 215, p. i-20, June 2008. 
Para a dimensão penetração bancária, o Sarma ${ }^{20}$ utilizou o indicador banco contas de depósito do Banco Mundial que inclui contas de depósito, poupança e depósitos a prazo, contas para empresas, indivíduos e outros. Já para a dimensão disponibilidade, foram tomados os dados em ramos de depósito em dinheiro dos bancos comerciais e outras instituições financeiras da mesma fonte. E finalmente para a dimensão de uso, foram utilizados os dados sobre créditos internos ao setor privado e residente e os dados sobre depósitos totais do International Financial Statistics (IFS, 2006) do FMI.

Um problema técnico encontrado por Sarma ${ }^{21}$ foi a disponibilidade de dados, pois, para uma investigação nas três dimensões estudadas por ele, as informações estavam disponíveis para apenas 55 países. Já ao restringir a dimensão para duas, ele encontrou dados comparáveis para uma amostra de 100 países. Na Tabela 1, são apresentados alguns resultados encontrados pelo autor.

Tabela 1 - Índice de Inclusão Financeira (IIF): 3 dimensões (2004)

\begin{tabular}{cccccc}
\hline País & Penetração & Acesso & Uso & IIF & Ranking \\
\hline Espanha & 0,651 & 1,000 & 0,706 & 0,737 & $1^{\text {o }}$ \\
Áustria & 1,000 & 0,568 & 0,619 & 0,667 & $2^{\text {o }}$ \\
Bélgica & 1,000 & 0,567 & 0,543 & 0,637 & $3^{\text {o }}$ \\
Dinamarca & 0,902 & 0,410 & 0,700 & 0,614 & $4^{\text {o }}$ \\
Brasil & $\mathbf{0 , 2 3 2}$ & $\mathbf{0 , 1 7 4}$ & $\mathbf{0 , 2 3 7}$ & $\mathbf{0 , 2 1 4}$ & $22^{\circ}$ \\
Índia & 0,164 & 0,075 & 0,269 & 0,166 & $29^{\circ}$ \\
Uganda & 0,019 & 0,000 & 0,025 & 0,015 & $54^{\circ}$ \\
Madagascar & 0,000 & 0,001 & 0,037 & 0,013 & $55^{\circ}$ \\
\hline
\end{tabular}

Fonte: Sarma ${ }^{22}$

O importante a se destacar é que os países apresentaram nível de inclusão bastante variado. Países como Espanha, Áustria, Bélgica e Dinamarca — nações mais desenvolvidas — possuem nível de inclusão financeira considerado alto. Por outro lado, países menos desenvolvidos como Brasil, Índia, Uganda e Madagascar apresentaram nível de inclusão financeira baixo.

$\mathrm{CNBV}^{23}$ realizou a primeira análise estatística com o objetivo de desenvolver indicadores de inclusão financeira no México. Esses indicadores foram apresentados em três categorias: os macroeconômicos, os de acesso e os de utilização. Para os primeiros, foram utilizados os empréstimos como percentual do PIB, e o quociente depósitos totais/PIB. Para os segundos, agências, caixas eletrônicos e terminais de pontos de venda por tipo geográfico e demográfico. Já para os de uso foram eleitos os relativos a crédito e ao cartão de crédito. Esses últimos foram demográficos nos níveis nacional, estadual e municipal.

O estudo realizado por $\mathrm{CNVB}^{24}$ proporcionou comparações da situação dos indicadores financeiros mexicano vis-à-vis aos de outros países desenvolvidos (como Austrália, Canadá, Reino Unido) e em desenvolvimento (Brasil, Rússia, Índia, China, África, etc.). Além disso, permitiu a construção do mapa da inclusão financeira do México nas suas dimensões internas: estado e município. Isso trouxe informações importantes para os formuladores de políticas daquele país, como por exemplo, de que a infraestrutura financeira está concentrada na sua região Centro-Oeste.

20 SARMA, M. Index of financial inclusion: a measure of financial sector inclusiveness. Working Paper, India, n. 215, p. i-20, June 2008.

21 SARMA, M. Index of financial inclusion: a measure of financial sector inclusiveness. Working Paper, India, n. 215, p. i-20, June 2008.

22 SARMA, M. Index of financial inclusion: a measure of financial sector inclusiveness. Working Paper, India, n. 215, p. i-20, June 2008.

23 COMISIÓN NACIONAL BANCARIA Y DE VALORES (NBV). Reporte de Inclusión Financiera 1. México, D.F., 2009.

24 COMISIÓN NACIONAL BANCARIA Y DE VALORES (NBV). Reporte de Inclusión Financiera 1. México, D.F., 2009. 
Comparando com o estudo de Sarma ${ }^{25}$, porém, a investigação do $\mathrm{CNVB}^{26}$ não busca a construção de um indicador único de inclusão financeira que permita comparar de forma geral a situação da inclusão financeira do México com a de outros países. Essa comparação é realizada indicador a indicador, o que torna o trabalho árduo e menos eficiente.

Chakravarty e $\mathrm{Pa}^{27}$ realizaram uma abordagem axiomática para medir a inclusão financeira na Índia em comparação com outros países selecionados. Na proposição de cálculo desses autores, baseado em análise de dados Cross-country e em nível subnacional, foi desenvolvido um quadro para agregar dados sobre inclusão financeira em diferentes dimensões. Com esse quadro os autores calcularam as contribuições percentuais de cada uma dessas dimensões, proporcionando um subsídio importante para os formuladores de políticas: a identificação das dimensões que são mais ou menos susceptíveis à inclusão global.

Com o quadro agregado desenvolvido por Chakravarty e $\mathrm{Pal}^{28}$, pôde-se verificar que para os países considerados de baixa renda há divergências no percentual de contribuição das dimensões de inclusão financeira estudadas, inclusive quando se compara essas nações. Para Madagascar, por exemplo, o indicador geográfico de penetração bancária é simplesmente zero. Já para Uganda, ele contribuiu com 15,4\% do valor do indicador de inclusão financeira.

Ao se comparar os países de renda média alta, na América Latina, tais como, Brasil, Argentina e Chile, percebe-se que para este último os indicadores demográficos de penetração bancária e de ATM responderam juntos por apenas $15,9 \%$ da inclusão financeira no país, enquanto que no Brasil e na Argentina eles contribuíram com, respectivamente, 26,29\% e 25,30\%. O interessante é que, na Bolívia, um país de renda média baixa do mesmo continente, esses indicadores demográficos foram responsáveis por 39,64\% do índice de inclusão financeira daquele país, valor considerado superior ao de nações de renda elevada como Bélgica (22,03\%) e Dinamarca (28,29\%).

Um ponto importante a se destacar refere-se ao trabalho Chakravarty e $\mathrm{Pal}^{29}$ que constitui-se em avanço nas pesquisas de mensuração da inclusão financeira ao medir as contribuições de cada dimensão no cálculo do índice de inclusão financeira, tarefa que $\mathrm{Sarma}^{30}$ não realizou. Portanto, aqueles autores além de constatar as divergências de nível de inclusão financeira entre os países, como o fizeram Sarma ${ }^{31}$ e CNBV ${ }^{32}$, eles evidenciaram quais dimensões tiveram participação significativa no IIF de cada um deles.

No Brasil, $\mathrm{BCB}^{33}$ calculou o Índice de Inclusão Financeira para os estados da federação, com relação aos anos de 2000, 2005 e 2010, utilizando-se da metodologia de cálculo do IIF adotada por Sarma e Pais (2010), com modificações no que diz respeito ao uso de variáveis e de suas dimensões. O Quadro 1 sintetiza as dimensões do IIF e os indicadores considerados em cada uma delas.

25 SARMA, M. Index of financial inclusion: a measure of financial sector inclusiveness. Working Paper, India, n. 215, p. i-20, June 2008.

26 COMISIÓN NACIONAL BANCARIA Y DE VALORES (NBV). Reporte de Inclusión Financiera 1. México, D.F., 2009.

27 CHAKRAVARTY, S. R.; PAL, R. Measuring financial inclusion: an axiomatic approach. Mumbai: Indira Gandhi Institute of Development Research, 2010. Disponível em: <http://www.igidr.ac.in/pdf/publication/WP-2010-003.pdf>. Acesso em: 01 jul. 2012.

28 CHAKRAVARTY, S. R.; PAL, R. Measuring financial inclusion: an axiomatic approach. Mumbai: Indira Gandhi Institute of Development Research, 2010. Disponível em: <http://www.igidr.ac.in/pdf/publication/WP-2010-003.pdf>. Acesso em: 01 jul. 2012. 29 CHAKRAVARTY, S. R.; PAL, R. Measuring financial inclusion: an axiomatic approach. Mumbai: Indira Gandhi Institute of Development Research, 2010. Disponível em: <http://www.igidr.ac.in/pdf/publication/WP-2010-003.pdf>. Acesso em: 01 jul. 2012. 30 SARMA, M. Index of financial inclusion: a measure of financial sector inclusiveness. Working Paper, India, n. 215, p. i-20, June 2008.

31 SARMA, M. Index of financial inclusion: a measure of financial sector inclusiveness. Working Paper, India, n. 215, p. i-20, June 2008.

32 COMISIÓN NACIONAL BANCARIA Y DE VALORES (NBV). Reporte de Inclusión Financiera 1. México, D.F., 2009.

33 BANCO CENTRAL DO BRASIL (BCB). Relatório de inclusão financeira. Brasília: BCB, 2011. 
Quadro 1 - Indicadores de Acesso e Uso

\begin{tabular}{|c|c|}
\hline Dimensão & Indicador \\
\hline $\begin{array}{l}\text { Acesso - } \\
\text { Disponibilidade } \\
\text { Geográfica } \\
\end{array}$ & $\begin{array}{c}\text { Agências por } 1.000 \mathrm{~km}^{2} ; \text { PAB, PAA e PAE por } \\
1.000 \mathrm{~km}^{2} \text {; Cooperativas por } 1.000 \mathrm{~km}^{2} ; \text { PAC por } \\
1.000 \mathrm{~km}^{2} \text {; Correspondentes por } 1.000 \mathrm{~km}^{2} .\end{array}$ \\
\hline $\begin{array}{l}\text { Acesso - } \\
\text { Disponibilidade } \\
\text { Demográfica }\end{array}$ & $\begin{array}{l}\text { Agências por } 10.000 \text { adultos; PAB por } 10.000 \text { adultos; } \\
\text { PAA por } 10.000 \text { adultos; PAE por } 10.000 \text { adultos; } \\
\text { Cooperativas por } 10.000 \text { adultos; PAC por } 10.000 \\
\text { adultos; Correspondentes por } 10.000 \text { adultos. }\end{array}$ \\
\hline Uso & $\begin{array}{l}\text { Razão Crédito/PIB; Credito (R } \$ \text { milhões) por } 1.000 \\
\text { adultos; Razão Depósitos/PIB; Depósitos (R } \$ \text { milhões) } \\
\text { por } 1.000 \text { adultos. }\end{array}$ \\
\hline
\end{tabular}

Fonte: $\mathrm{BCB}^{34}$

Os resultados encontrados pelo $\mathrm{BCB}^{35}$ indicam que há uma forte diferença no grau de inclusão financeira dos estados brasileiros, sendo que os das regiões sul, sudeste e centro-oeste apresentaram grau de inclusão financeira superior aos da região norte e nordeste. O estudo também evidenciou que o IIF foi crescente nos três períodos em análise, comprovando que o país elevou o seu nível de inclusão financeira na última década. Essa mesma dinâmica foi evidenciada para cada estado da federação.

$\mathrm{O}$ estudo do $\mathrm{BCB}^{36}$ trouxe uma contribuição importante no comparativo com os textos já analisados: mensurar a inclusão financeira ao longo de três períodos de tempo. Com isso pode evidenciar que o grau de inclusão financeira no Brasil e em todas as suas unidades da federação cresceu. Sua pesquisa, porém, não permitiu verificar qual foi a contribuição percentual de cada uma das dimensões da inclusão financeira, como o fez Chakravarty e $\mathrm{Pal}^{37}$, conforme já discutido alguns parágrafos acima.

\subsection{A inclusão financeira no Brasil}

O ponto de partida para a formulação da concepção de inclusão financeira no Brasil foi possível a partir das discussões acerca do microcrédito e, posteriormente, das microfinanças. Esse debate foi ganhando contornos mais bem definidos, a ponto de ser tratado como inclusão financeira, ao qual foi denominado pelo BCB.

A inclusão financeira é conceituada pelo $\mathrm{BCB}^{38}$ como - "o processo de efetivo acesso e uso pela população de serviços financeiros adequados às suas necessidades, contribuindo com sua qualidade de vida". Essa definição foi aperfeiçoada desde a sua primeira versão e ela aponta para a necessidade do sistema financeiro em atender indistintamente a população com serviços apropriados, visto que há carências tanto na oferta como na qualidade de serviços para o público de menor renda e para os pequenos negócios produtivos no Brasil.

É importante destacar que, embora o tema tenha ganhado maior fôlego nos últimos anos, os passos iniciais para o debate sobre a inclusão financeira ocorreu no governo de Fernando Henrique Cardoso, em meados da década de 1990. Nesse momento, o BCB já começava o processo de avaliação e estudos visando o aumento da oferta de serviços financeiros para populações de baixa renda, considerado elemento de fundamental importância para o combate à desigualdade social no país.

No início do milênio, o BCB aprimora os canais de acesso ao sistema financeiro e a sua regulamenta-

34 BANCO CENTRAL DO BRASIL (BCB). Relatório de inclusão financeira. Brasília: BCB, 2011.

35 BANCO CENTRAL DO BRASIL (BCB). Relatório de inclusão financeira. Brasília: BCB, 2011.

36 BANCO CENTRAL DO BRASIL (BCB). Relatório de inclusão financeira. Brasília: BCB, 2011.

37 CHAKRAVARTY, S. R.; PAL, R. Measuring financial inclusion: an axiomatic approach. Mumbai: Indira Gandhi Institute of Development Research, 2010. Disponível em: <http://www.igidr.ac.in/pdf/publication/WP-2010-03.pdf>. Acesso em: 01 jul. 2012.

38 BANCO CENTRAL DO BRASIL (BCB). Relatório de inclusão financeira. Brasília: BCB, 2011. 
ção, sobretudo estimula a expansão dos correspondentes no país - empresas contratadas por instituições financeiras para prestar determinada gama de serviços em nome e sob responsabilidade da instituição contratante. O resultado dessa iniciativa é que todos os municípios brasileiros passaram a ter algum canal de prestação de serviço financeiro, quer seja por intermédio de dependências de instituições financeiras, quer seja por correspondentes. $\mathrm{O}$ avanço normativo permitiu que os correspondentes fossem contratados para atuarem em qualquer praça, acentuando as vantagens desse tipo de canal para ampliar o acesso e uso de serviços financeiros pela população.

Em 2009, o BCB organizou um conjunto de estudos acerca da Inclusão Financeira, cujo propósito foi reunir diferentes perspectivas de atores na realização de diagnóstico do setor de microfinanças no Brasil. O documento identifica os pontos fortes e fragilidades das microfinanças, a fim consubstanciar a tomada de decisão no que tange aos projetos e parceiras para promoção da inclusão financeira do brasileiro.

Foi realizado o I Fórum Banco Central sobre Inclusão Financeira, que teve como propósito a consolidação de diagnóstico sobre microfinanças no Brasil e promoção de parcerias. Em 2010 foi construído o Relatório de Inclusão Financeira n ${ }^{\circ} 1$ do BCB, que reúne informações sobre acesso e uso de serviços financeiros, a partir de dados de instituições reguladas. Em seguida, ocorreu o II Fórum Banco Central sobre Inclusão Financeira, que propiciou a construção de agenda para estudo e aprimoramento do marco regulatório. Em 2011, o BCB publicou o Relatório de Inclusão Financeira n 2, cujo conteúdo apresenta um aprimoramento em relação ao RIF n ${ }^{\circ} 1$. O documento, mais minucioso, exibe um estudo por segmento e integração de dados de acesso e de uso. Ao final do ano de 2011 a instituição realizou o III Fórum BC sobre Inclusão Financeira, cujo propósito foi buscar a estruturação e lançamento da Parceria Nacional para Inclusão Financeira.

De acordo com $\mathrm{BCB}^{39}$, entre 2000 e 2010, o Brasil passou de 19.000 para 150.000 correspondentes que cobrem cada um dos municípios. Os serviços prestados por esses pontos referem-se a pagamento de contas, depósitos, saques e transferências de dinheiro. E tem sido um canal largamente utilizado em favor do pagamento dos programas de transferência de renda do governo federal, a exemplo do Programa Bolsa Família (PBF).

A vantagem dos correspondentes é que, dada as dimensões continentais do Brasil, muitas delas de difícil acesso, com pequena população e, na maioria dos casos, de baixa renda, permitem superar os diversos empecilhos para a expansão dos pontos de atendimento, tais como instalação em locais em que a escala de negócios é reduzida ${ }^{40}$. Em síntese, os custos de operação dos correspondentes são inferiores aos das tradicionais agências bancárias.

Com a devida utilização dos conceitos microeconômicos, é possível inferir que, na medida em que se estabelece a necessidade de expansão da rede bancária (agências) para localidades de baixa renda e baixa densidade populacional, a menor densidade de uso dos serviços financeiros, quando comparadas a regiões urbanas, representa uma relação receita/custo marginal inferior, sendo mais rentável para os oligopólios bancários ampliar a rede em localidades de maior concentração populacional, como as regiões metropolitanas. Portanto, as especificidades econômicas e estruturais dos correspondentes em relação às agências bancárias (tradicionais) permitem sua construção em locais de pequena população e baixa renda.

Outro importante canal de acesso ao sistema financeiro tem se difundindo, são às cooperativas de crédito, especialmente no que tange ao crédito rural e associações produtivas, que a partir da década de 1990, foram fortalecidas do ponto de vista de uma regulação mais clara e objetiva. As cooperativas de crédito cumprem a função de promover a aplicação de recursos privados e públicos, assumindo os riscos corres-

39 BANCO CENTRAL DO BRASIL (BCB). Plano de ação para fortalecimento do ambiente institucional. Brasília: BCB, 2012. 40 BANCO CENTRAL DO BRASIL (BCB). Perspectivas e desafios para inclusão financeira no Brasil: visão de diferentes atores. In: FELTRIN, Luiz (Coord.); VENTURA, Elvira Cruvinel Ferreira (Coord.); DODL, Alessandra Von Borowski (Coord.). Projeto inclusão financeira. Brasília: BCB, 2009. 
pondentes em favor da comunidade onde se inserem ${ }^{41}$

Com base nos dados disponibilizados pelo $\mathrm{BCB}^{42}$, entre 2000 e 2010, o número de pontos de acesso de cooperativas de crédito aumentou de 2,6 para 6,4 mil e o número de cooperados, de 1,5 para 5,1 milhões. As cooperativas de crédito estão presentes em $40 \%$ dos municípios brasileiros e em $25 \%$ dos municípios sem agência bancária. Conforme a Tabela 2, nota-se que de 2006 para 2010 os diversos formatos de pontos de atendimento aumentaram substancialmente, sobretudo os PAAs, ATMs, POSs.

Tabela 2 - Número de pontos de atendimento por canal de acesso (2006 e 2010)

\begin{tabular}{cccc}
\hline Canal de Acesso & \multirow{2}{*}{$\mathbf{2 0 0 6}$} & $\mathbf{2 0 1 0}$ & $\begin{array}{c}\text { Crescimento } \\
\text { \% }\end{array}$ \\
\hline Agências bancárias & 18.087 & 19.813 & 9,54 \\
Pontos de correspondente & 101.038 & 151.623 & 50,07 \\
Cooperativas singulares e PACs & 3.819 & 4.577 & 19,85 \\
Posto de Atendimento Bancário (PAB) & 6.599 & 6.678 & 1,20 \\
Posto Avançado de Atendimento (PAA) & 406 & 1.978 & 387,19 \\
Posto de Atendimento Bancário Eletrônico (PAE) & 29.865 & 35.498 & 18,86 \\
AutomatedTellerMachine (ATM) & 97.780 & 174.920 & 78,89 \\
Point ofS Sale (POS) & 1.845 .064 & 3.398 .890 & 84,22 \\
\hline
\end{tabular}

Fonte: $\mathrm{BCB}^{43}$

O conjunto de esforços e os resultados até então identificados constituem avanços expressivos no processo de inclusão financeira da população. Ademais, as dificuldades de parte da população e das Micro e Pequenas Empresas (MPEs) são latentes no que se refere ao acesso permanente de serviços financeiros. Isso constitui entrave para elevar o bem-estar, alavancar as atividades econômicas e propiciar maiores oportunidades de geração de emprego e renda para a população. Para as MPEs, os entraves para o efetivo acesso ao sistema financeiro nacional estão relacionados aos riscos, à precariedade de suas informações contábeis e aos pequenos volumes de recursos envolvidos.

\subsection{O papel das microfinanças para a inclusão financeira}

$\mathrm{Na}$ literatura econômica, observa-se que as microfinanças são um dos principais mecanismos para a inclusão financeira e para o desenvolvimento econômico e social. As microfinanças ganharam repercussão mundial por meio da visão estratégica de Muhammad Yunus, que através do Grameen Bank conquistou interesses públicos e privados na promoção dessa modalidade de negócio, alcançando mais de 160 milhões de clientes pelo mundo.

Hulme ${ }^{44}$ explica que os créditos concedidos pelo Grameen Bank são destinados para diversos fins, quais sejam: negócio, bens de consumo, saúde, educação e até pagamento de dote. Do ponto de vista do investimento, os créditos não eram destinados exclusivamente para os micronegócios. Ao invés disso, eles faziam parte de portfólios financeiros complexos das famílias de baixa renda.

O microcrédito tornou-se uma ferramenta usada na luta contra a pobreza, a partir do entendimento de que por meio do encorajamento, da geração de autonomia e estímulo do espírito empreendedor entre os

41 ZIGER, V. Cooperativismo de crédito solidário: inclusão social e desenvolvimento local. In: FELTRIM, L. E.; VENTURA, E. C. F.; DODL, A. V. B. (Coord.). Perspectivas e desafios para inclusão financeira no Brasil: visão de diferentes atores. Brasília: Banco Central do Brasil, 2009.

42 BANCO CENTRAL DO BRASIL (BCB). Plano de ação para fortalecimento do ambiente institucional. Brasília: BCB, 2012.

43 BANCO CENTRAL DO BRASIL (BCB). Relatório de inclusão financeira. Brasília: BCB, 2011.

44 HULME, David. The story of the grameen bank: from subsised microcredit to market-based microfinance. Manchester: Brooks World Poverty Institute: 2008. (BWPI. Working paper, 60). 
mais pobres é possível promover sua emancipação e superar a pobreza ${ }^{45}$. As microfinanças, portanto, são ferramentas de enfrentamento da pobreza, que contribuem para a promoção do desenvolvimento humano, na medida em que fornecem os meios para a superação da pobreza e, por outro lado ampliam novos consumidores de crédito, elevando a demanda para o consumo de bens e serviços.

O processo de inclusão financeira, especialmente a expansão do crédito, permite a aquisição de bens de consumo, o que tem grande importância por permitir acréscimos na demanda, e ao tempo aumenta o consumo, eleva o emprego, melhorando os indicadores econômicos e sociais.

A recente inclusão de camadas de baixa renda no mercado de bens e no sistema financeiro brasileiro ampliou a demanda por bens de consumo e correspondeu de algum modo, durante a crise 2009, num importante mecanismo anticíclico de sustentação da demanda e do crescimento ${ }^{46}$.

Experiências como a da Bolívia, que, embora seja um país pobre, com grande extensão geográfica e reduzida densidade populacional, alcançou resultados positivos do ponto de vista econômico e social, e levou sua nação a possuir um dos mercados de microfinanças mais desenvolvido do mundo ${ }^{47}$. Esse resultado, entre outros fatores, se deve as ações voltadas para o fomento e disseminação das microfinanças, o que passa essencialmente por uma política mais ampla de inclusão financeira.

$\mathrm{Na}$ mesma direção, Coutinho, Ambrozio, Sant'Anna e Montoro ${ }^{48}$, atentos às transformações que vêm ocorrendo no sistema financeiro, destacam que, as microfinanças desempenham papel essencial para que se dê, de fato, a inclusão financeira. $\mathrm{Na}$ perspectiva desses autores, as microfinanças envolve disponibilidade de distintos serviços financeiros, sobretudo a disponibilidade de crédito em pequena escala, haja vista que a oferta de microcrédito assume papel significativo na superação das restrições de crédito da população de baixa renda. A ideia é que o acesso a essa modalidade de crédito possibilita as pessoas com elevada restrição orçamentária a realizar lucros com projetos de sucesso, ampliando sua renda e se emancipando socialmente.

No Brasil, ainda em menor grau, as microfinanças vêm ganhando destaque. Somado aos demais canais de acesso, as microfinanças potencializam as alternativas de ampliação do sistema financeiro. As microfinanças se orientam para o desenvolvimento de produtos e serviços financeiros adequados e acessíveis à população excluída do tradicional sistema financeiro ${ }^{49}$. A modalidade microcrédito está entre os diversos produtos microfinanceiros, que além de servir para os grupos sociais menos favorecidos, constitui num importante instrumento de financiamento das MPEs.

Segundo Santos, Nogueira e Moreno ${ }^{50}$, uma operação de microcrédito não é viável através da metodologia de crédito bancário tradicional. Nitsch e Santos ${ }^{51}$ explicam que, os critérios de análise de risco, contrata-

45 O impacto das microfinanças, segundo o Banco Mundial, pode ser percebido na redução da pobreza e fome, na educação básica universal, promoção equidade de gênero e empoderamento de mulheres, redução da mortalidade infantil e melhora significativa na saúde materno infantil. SEBRAE. Inclusão financeira: empreendedores individuais estão nos radares de bancos, instituições microfinanceiras e cooperativas de créditos. Conbecer Sebrae pequenos negócios \& finanças, Brasília, n. 12, p. 1-52, maio 2010.

46 BRUSKY, B.; FORTUNA, J. P. Entendendo a demanda para as microfinanças no Brasil: um estudo qualitativo em duas cidades. 2002. Disponível em: <http://www.bndes.gov.br/SiteBNDES/export/sites/default/bndes_pt/Galerias/Arquivos/conhecimento/microfin/01livreto.pdf> Acesso em: 01 set. 2013.

47 PRADO, F. Experiência boliviana. In: SEMINÁRIO BNDES MICROFINANÇAS: ENSAIOS E EXPERIÊNCIAS, 2000, Rio de Janeiro. Anais ... Rio de Janeiro: BNDES, 2000.

48 COUTINHO, L. G.; AMBROZIO, A. M. H. P.; SANT'ANNA, A. A.; MONTORO, G. C. F. Inclusão financeira no Brasil: o papel do BNDES. In: FELTRIM, Luiz Edson; VENTURA, Elvira Cruvinel Ferreira; DODL, Alessandra von Borowski. Perspectivas e desafios para inclusão financeira no Brasil: visão de diferentes atores. Projeto inclusão financeira. Brasília: BCB, 2009.

49 SANTOS, C. A.; NOGUEIRA, D. G.; MORENO, E. Microfinanças, microcrédito e a oferta de serviços financeiros para os microempreendimentos. In: SANTOS, Carlos Aberto dos (Coord.). Sistema financeiro e as micro e pequenas empresas: diagnósticos e perspectivas. 2. ed. Brasília: SEBRAE, 2004.

50 SANTOS, C. A.; NOGUEIRA, D. G.; MORENO, E. Microfinanças, microcrédito e a oferta de serviços financeiros para os microempreendimentos. In: SANTOS, Carlos Aberto dos (Coord.). Sistema financeiro e as micro e pequenas empresas: diagnósticos e perspectivas. 2. ed. Brasília: SEBRAE, 2004.

51 NITSCH, M.; SANTOS, C. A. Da repressão financeira ao microcrédito: notas sobre princípios e alternativas institucionais para a expansão da fronteira financeira. Revista de economia Política, São Paulo, v. 21, n. 84, p. 172-183, trimestral, 2001. 
ção, concessão, monitoramento, pagamento e recuperação do microcrédito não podem ser os mesmos das operações de crédito voltadas para assalariados e empresas de porte.

$\mathrm{Na}$ literatura sobre microcrédito não foi identificado um consenso acerca de sua definição. Ademais, no Brasil, por meio da interpretação da Lei no 10.735/2003 pode-se construir uma noção do significado do microcrédito. Partindo da percepção que há baixa disponibilidade de recursos para operações de crédito para a população de baixa renda e microempreendedores, a lei obriga os bancos múltiplos com carteira comercial e os bancos comerciais a aplicarem uma parcela dos depósitos à vista em operações para a população de baixa renda e microempreendedores. Entende-se, portanto, que a modalidade de microcrédito está direcionada, essencialmente, para a população de baixa renda e para as MPEs.

Embora não seja objetivo do presente trabalho avaliar as políticas de microcrédito no Brasil, cabe destacar as principais políticas de fomento ao crédito para a população de baixa renda, tais como o Programa Nacional de Fortalecimento da Agricultura Familiar (PRONAF), o Programa Nacional de Microcrédito Produtivo Orientado (PNMPO) para as MPEs, Programa Minha Casa Minha Vida (PMCMV), entre outros. O PRONAF mobiliza recursos para o custeio da lavoura e investimentos no setor produtivo rural. O PNMPO, instituído pelo Ministério do Trabalho e Emprego, tem o objetivo de incentivar a geração de trabalho e renda entre os microempreendedores populares. O PMCMV incentiva e financia à produção e à aquisição de novas unidades habitacionais pelas famílias com renda mensal de até dez salários mínimos, que residam em qualquer dos municípios brasileiros.

\section{Metodologia}

A metodologia do presente trabalho está orientada a partir de um esquema explicativo, que contempla dois níveis de análise: a exposição do IIF para os estados do Nordeste em três recortes (2000, 2005 e 2010); e a quantificação da correlação entre o IIF e o IFDM dos estados do Nordeste.

Os valores do IIF foram coletados no Relatório de Inclusão Financeira elaborado pelo BCB B2 $^{52}$ Já os valores do Índice FIRJAN de Desenvolvimento Municipal (IFDM) foram catalogados no site do FIRJAN, que também disponibiliza o resultado por estado.

O Sistema FIRJAN acompanha o desenvolvimento de todos os 5.564 municípios brasileiros em três áreas: Emprego \& Renda, Educação e Saúde. Ele é feito, exclusivamente, com base em estatísticas públicas oficiais, disponibilizadas pelos ministérios do Trabalho, Educação e Saúde. O índice varia de 0 a 1, sendo que, quanto mais próximo de 1, maior será o nível de desenvolvimento.

No que se refere à quantificação da correlação, é montada uma base de dados do IIF para cada estado do Nordeste nos anos de 2000, 2005 e 2010. Para o IFDM utilizam-se os anos de 2000, 2005 e 2009, sendo este último tido como uma aproximação de 2010, tendo em vista que este ano ainda não foi divulgado. Essa correlação é calculada por meio do programa operacional Microsoft Office Excel. Os resultados são organizados em uma Tabela de forma que garanta a visualização das correlações tanto entre o IIF e IFDM agregado como entre o IIF e o IFDM (Emprego \& Renda), IFDM (Educação) e IFDM (Saúde).

\section{Análise de resultados}

Com vistas a avaliar o grau de inclusão financeira nos estados do Nordeste brasileiro, utilizam-se os resultados obtidos pelo IIF do BCB. Embora o IIF seja um indicador sintético, ele é capaz de mostrar tendências

52 BANCO CENTRAL DO BRASIL (BCB). Relatório de inclusão financeira. Brasília: BCB, 2011. 
gerais, permitindo verificar o nível de inclusão dos estados nordestinos em uma escala numérica.

Na tabela 3, apresenta-se o IIF dos estados do Nordeste para os anos de 2000, 2005 e 2010, assim como o seu respectivo ranking. A primeira evidência que se extrai da leitura da Tabela é que, no período em análise, o IIF cresceu para todos os estados nordestinos, exceto o do Piauí, que teve um decrescimento do IIF em 2005 com relação a 2000, porém, em 2010, o seu índice duplicou. Em média, o Nordeste saltou de 5,02 em 2000 para 13,37 em 2010.

Tabela 3 - Índice de Inclusão Financeira dos Estados do Nordeste Brasileiro

\begin{tabular}{ccccccc}
\hline \multirow{2}{*}{ Estados } & \multicolumn{2}{c}{2000} & \multicolumn{2}{c}{2005} & \multicolumn{2}{c}{2010} \\
\cline { 2 - 6 } & Valor & Posição & Valor & Posição & Valor & Posição \\
\hline Sergipe & 7,4 & 1 & 11 & 1 & 16,0 & 1 \\
Pernambuco & 3,5 & 8 & 8,7 & 2 & 15,6 & 2 \\
Rio Grande do & 5,6 & 3 & 8,4 & 4 & 14,8 & 3 \\
Norte & 4,5 & 6 & 7,8 & 6 & 14,7 & 4 \\
Paraíba & 5,4 & 4 & 8,6 & 3 & 14,4 & 5 \\
Alagoas & 5,3 & 5 & 7,7 & 7 & 13,9 & 6 \\
Bahia & 4,4 & 7 & 8,0 & 5 & 12,3 & 7 \\
Ceará & 6,6 & 2 & 5,0 & 8 & 10,1 & 8 \\
Piauí & 2,5 & 9 & 4,2 & 9 & 8,5 & 9 \\
Maranhão & & & & & &
\end{tabular}

Fonte: $\mathrm{BCB}^{53}$

Destaca-se que, infelizmente, o BCB não disponibiliza dados acerca dos indicadores que compõe o IIF por estado. De qualquer modo, é possível levantar a hipótese de que parte do aumento do IIF nos estados nordestinos se deve às medidas de acesso, sobretudo ao número de correspondentes.

Em termos de disponibilidade (geográfica e demográfica), os correspondentes cresceram substancialmente no Brasil. Considerando que o resultado dos desvios apresentou grande dispersão em relação às unidades da federação e que há forte concentração dos pontos de correspondentes nos estados do Sudeste e Sul ${ }^{54}$. Supõe-se que os acréscimos do número de correspondentes nos estados do Nordeste na última década têm sustentado a maior expansão do IIF. Entretanto, a região, em si, agrega em maior número, parte dos estados mais deficitários em termos do IIF, formando, portanto, os sujeitos alvo das políticas de inclusão financeira.

Conforme a apresentação das posições dos estados nordestinos no ranking regional, calculado a partir do valor obtido no IIF, o grande destaque é para o estado de Sergipe que se manteve na primeira posição nos três períodos exibidos. Já o estado do Maranhão, por sua vez, manteve-se na última posição.

Outro ponto a se destacar é que a razão entre o maior e o menor valor do índice é declinante ao longo do período. Em 2000, essa razão foi de 2,96; em 2005, de 2,62; e em 2010, de 1,88. Assim, pode-se inferir que as diferenças de inclusão financeira entre os estados nordestinos estão se reduzindo, ainda que de forma lenta.

Em termos absolutos, porém, a diferença é crescente, 4,9; 6,8 e 7,5, respectivamente, 2000, 2005 e 2010. O estado de Pernambuco foi o que apresentou maior crescimento absoluto, o que lhe garantiu saltar da penúltima posição em 2000 para a segunda posição em 2010. O estado da Paraíba também exibiu uma melhora no seu IIF. Já o estado do Piaú foi o que apresentou menor crescimento absoluto, ao passo que perdeu a posição de segundo colocado em 2000 para a oitava posição em 2010.

Um ponto importante para se destacar neste momento é que a inclusão financeira, apontada pelo IIF, ocorreu de forma desigual entre os estados nordestinos, demonstrando com isso as desigualdades intrínse-

53 BANCO CENTRAL DO BRASIL (BCB). Relatório de inclusão financeira. Brasília: BCB, 2011.

54 BANCO CENTRAL DO BRASIL (BCB). Relatório de inclusão financeira. Brasília: BCB, 2011. 
cas da própria região. Esse argumento é comprovado por meio do resultado do desvio-padrão, pois nota-se que este se eleva consideravelmente ao longo dos períodos em análise: 1,50; 2,03 e 2,56.

$\mathrm{Na}$ sequência é analisada a correlação linear entre o IIF e o IFDM dos estados nordestinos. O intuito dessa análise surge da curiosidade de quantificar a força de suas relações e de se verificar empiricamente se as duas variáveis variam positivamente para cada estado.

A Tabela 4 exibe o resultado dessa correlação para cada estado da região Nordeste, classificada em ordem decrescente de correlação com o IFDM. A primeira observação é de que há uma forte correlação positiva entre o IIF e os indicadores de bem-estar do IFDM para todos os estados nordestinos. Com exceção da Bahia e do Piauí, cujas correlações foram respectivamente de 0,856 e 0,762 , os demais estados tiveram a correlação superior a 0,9 .

Tabela 4 -Correlação do IFF com os Indicadores de Bem-Estar

\begin{tabular}{lllll}
\hline Estado & IFDM & IFDM-E\&R & IFDM-E & IFDM-S \\
\hline Ceará & 1,000 & 1,000 & 0,973 & 0,980 \\
Maranhão & 0,995 & 0,706 & 0,907 & 0,999 \\
Pernambuco & 0,993 & 0,983 & 0,986 & 1,000 \\
Paraíba & 0,958 & 0,944 & 0,931 & 0,974 \\
Alagoas & 0,954 & $(0,061)$ & 0,990 & 0,983 \\
Rio Grande do Norte & 0,901 & 0,356 & 0,938 & 0,996 \\
Sergipe & 0,901 & 0,797 & 0,930 & 0,966 \\
Bahia & 0,856 & 0,573 & 0,938 & 0,996 \\
Piauí & 0,762 & 0,879 & 0,642 & 0,822 \\
\hline
\end{tabular}

Fonte: Elaboração Própria

Ao se observar a correlação do IFF com os três componentes do IFDM (Emprego \& Renda, Educação e Saúde), verifica-se que existe uma forte correlação positiva entre os mesmos, com algumas poucas exceções, são elas: IFDM-E\&R para os estados da Alagoas $(-0,061)$ e Rio Grande do Norte $(0,356)$. Essas ressalvas são explicadas pela queda apresentada nos indicadores FIRJAN para Emprego \& Renda para estes estados em 2009.

A hipótese que se pode elaborar da correlação positiva, entre o IIF e os indicadores de bem-estar, consiste no fato deque maior inclusão financeira pode causar maior desenvolvimento da economia do nordeste brasileiro, induzindo melhores níveis de bem-estar na região, medidos pelos indicadores FIRJAN. Por tratar-se de uma correlação, pode-se inferir, ainda, que melhoras nos níveis de bem-estar da sociedade nordestina induzirá a maior grau de inclusão financeira de sua população.

\section{Conclusão}

A literatura indica evidências científicas da importância do setor financeiro para o desenvolvimento econômico de um país, uma vez que os serviços financeiros proporcionam melhoria do bem-estar de sua população. Por outro lado, é necessário fazer com que os benefícios de um sistema financeiro sejam experimentados por todos os indivíduos, já que isso quase sempre não ocorre devido às desigualdades socioeconômicas que cada sociedade apresenta.

Para mitigar essa situação de desigualdade, é necessária à implantação de políticas públicas específicas para esse fim. E para isso torna-se necessário ter um retrato o mais próximo possível da realidade para a ideal interferência dos organismos públicos. No que diz respeito à inclusão dos indivíduos ao sistema financeiro, é preciso se ter uma radiografia do mapa da inclusão financeira. 
A literatura consultada para a presente pesquisa revela um pouco dessa radiografia com relação ao tema: a inclusão financeira. O que se viu foi que, mesmo ao se utilizar metodologias diferenciadas de mensuração dessa forma de inclusão, o que se constatou foi que os países apresentam grau de inclusão financeira diferenciada, seja entre regiões com níveis de renda diferenciados, seja com nações com o mesmo nível de renda. Inclusive nos espaços subnacionais essa divergência é percebida, como foi visto no caso do México e do Brasil.

Com relação à região do nordeste brasileiro, foco do presente estudo, o que se constatou foi que essa parte do Brasil experimentou forte crescimento do nível de inclusão financeira na última década, embora a região apresente-se em uma situação desfavorável no comparativo com as regiões sul, sudeste e centro-oeste.

Verificou-se, também, que há diferenças no grau de inclusão financeira entre os estados dessa região o que demandará dos formuladores de políticas a adoção de medidas inclusivas diferenciadas.

Constatou-se, ainda, que, no cálculo de correlação entre os IIF e os índices da FIRJAN, há uma forte correlação positiva entre os mesmos. Isso leva à hipótese de que a inclusão financeira experimentada pelos estados nordestinos pode ter contribuído para desenvolvimento econômico da região e consequentemente de sua população. O contrário também pode ser proposto, que o desenvolvimento econômico da região nordeste pode ter elevado o nível de inclusão da região.

Por último convém destacar que a ausência de dados específicos dos componentes do IIF para os estados nordestinos constituiu-se num empecilho para a adequada avaliação da inclusão financeira nessas unidades federativas, restringindo a análise dos percentuais de contribuição de cada dimensão da inclusão financeira. Abrindo, portanto, uma janela para investigações futuras.

\section{REFERÊNCIAS}

ARESTIS, P.; DEMETRIADES, P. Finance and growth: is Schumpeter right? Análise Econômica, Porto Alegre, v. 6, n. 30, p. 5-21, semestral, 1998.

BANCO CENTRAL DO BRASIL (BCB). Perspectivas e desafios para inclusão financeira no Brasil: visão de diferentes atores. In: FELTRIN, Luiz (Coord.); VENTURA, Elvira Cruvinel Ferreira (Coord.); DODL, Alessandra Von Borowski (Coord.). Projeto inclusão financeira. Brasília: BCB, 2009.

BANCO CENTRAL DO BRASIL (BCB). Plano de ação para fortalecimento do ambiente institucional. Brasília: BCB, 2012.

BANCO CENTRAL DO BRASIL (BCB). Relatório de inclusão financeira. 2010. Disponível em: <http:/ /www. bcb.gov.br/Nor/relincfin/relatorio_inclusao_financeira.pdf>. Acesso em: 06 jul. 2012.

BANCO CENTRAL DO BRASIL (BCB). Relatório de inclusão financeira. Brasília: BCB, 2011.

BRUSKY, B.; FORTUNA, J. P. Entendendo a demanda para as microfinanças no Brasil: um estudo qualitativo em duas cidades. 2002. Disponível em: < http://www.bndes.gov.br/SiteBNDES/export/sites/default/bndes_pt/Galerias/Arquivos/conhecimento/microfin/01livreto.pdf> Acesso em: 01 set. 2013.

CHAKRAVARTY, S. R.; PAL, R. Measuring financial inclusion: an axiomatic approach. Mumbai: Indira Gandhi Institute of Development Research, 2010. Disponível em: <http://www.igidr.ac.in/pdf/publication/WP2010-003.pdf>. Acesso em: 01 jul. 2012.

COMISIÓN NACIONAL BANCARIA Y DE VALORES (NBV). Reporte de Inclusión Financiera 1. México, D.F., 2009. 
COUTINHO, L. G.; AMBROZIO, A. M. H. P.; SANT’ANNA, A. A.; MONTORO, G. C. F. Inclusão financeira no Brasil: o papel do BNDES. In: FELTRIM, Luiz Edson; VENTURA, Elvira Cruvinel Ferreira; DODL, Alessandra von Borowski. Perspectivas e desafios para inclusão financeira no Brasil: visão de diferentes atores. Projeto inclusão financeira. Brasília: BCB, 2009.

DARRAT, A. F. Are financial deepening and economic growth causality related?: another look at the evidence. International Economic Journal, Seoul, v. 13, n. 3, p. 19-35, autumn 1999.

DEMETRIADES, P. O.; HUSSEIN, K. A. Does financial development cause economic growth?: time series evidence from 16 countries. Journal of Development Economics, Republic of Korea, n. 51, p. 387-411, quarterly, 1996.

FIGUEIREDO, Odail. Inclusão financeira: conquistas. Revista Rumos, Brasília, n. 52, p. 52-55, maio/jun. 2012.

GREENWOOD, J.; JOVANOVIC, B. Financial development, growth, and the distribution of income. Journal of Political Economy, CHicago, v. 98, n. 5, part 1, p. 1076-1107, quarterly, 1990.

HANNIG, A.; JANSEN, S. Financial inclusion and financial stability: current policy issues. ADBI Working Paper, Tokyo, n. 259, Dec. 2010. Disponível em: <http://www.adbi.org/working-paper/2010/12/21/4272. financial.inclusion.stability.policy.issues/>. Acesso em: 01 jul. 2012.

HULME, David. The story of the grameen bank: from subsised microcredit to market-based microfinance. Manchester: Brooks World Poverty Institute: 2008. (BWPI. Working paper, 60).

KHAN, M. S.; SENHADJI, A. S. Threshold effects in the relationship between inflation and growth. IMF Working Paper WP/00/110, Washington, June 2000.

LEE, B. Financial development and economic growth: the role of information accumulation. Economic Papers, Australia, v. 4, n. 1, p. 1-39, quarterly, 2001.

LEE, J. Financial development by learning. Journal of Development Economics, Republic of Korea, n. 50, p. 147-64, quarterly, 1996.

LEVINE, R. Financial development and economic growth: views and agenda. Journal of Economic Literature, Nashville, n. 35, p. 688-726, quarterly, 1997.

MATOS, O. C. Inter-relações entre desenvolvimento financeiro, exportações e crescimento econômico: análise da experiência brasileira. Notas Técnicas do Banco Central do Brasil, Brasília, n. 40, out. 2003.

MODIGLIANI, F.; MILLER, M. H. The cost of capital, corporation finance and the theory of investment. American Economic Review, Pittsburgh, n. 48, p. 261-97, monthly, 1958.

NITSCH, M.; SANTOS, C. A. Da repressão financeira ao microcrédito: notas sobre princípios e alternativas institucionais para a expansão da fronteira financeira. Revista de economia Política, São Paulo, v. 21, n. 84, p. 172-183, trimestral, 2001.

PEARCE, D. Financial Inclusion in the Middle East and North Africa: analysis and roadmap recommendations. Middle East and North Africa Region: The World Bank, 2011. (Policy Research Working Paper, 5610).

PRADO, F. Experiência boliviana. In: SEMINÁRIO BNDES MICROFINANÇAS: ENSAIOS E EXPERIÊNCIAS, 2000, Rio de Janeiro. Anais ... Rio de Janeiro: BNDES, 2000.

SANTOS, C. A.; NOGUEIRA, D. G.; MORENO, E. Microfinanças, microcrédito e a oferta de serviços financeiros para os microempreendimentos. In: SANTOS, Carlos Aberto dos (Coord.). Sistema financeiro e as micro e pequenas empresas: diagnósticos e perspectivas. 2. ed. Brasília: SEBRAE, 2004.

SARMA, M. Index of financial inclusion: a measure of financial sector inclusiveness. Working Paper, India, n. 215, p. i-20, June 2008. 
SCHUMPETER, J. A. A teoria do desenvolvimento econômico. São Paulo: Abril Cultural, 1982.

SEBRAE. Inclusão financeira: empreendedores individuais estão nos radares de bancos, instituições microfinanceiras e cooperativas de créditos. Conhecer Sebrae pequenos negócios \& finanças, Brasília, n. 12, p. 1-52, maio 2010.

ZIGER, V. Cooperativismo de crédito solidário: inclusão social e desenvolvimento local. In: FELTRIM, L. E.; VENTURA, E. C. F.; DODL, A. V. B. (Coord.). Perspectivas e desafios para inclusão financeira no Brasil: visão de diferentes atores. Brasilia: Banco Central do Brasil, 2009. 
Para publicar na revista Brasileira de Políticas Públicas, acesse o endereço eletrônico www.rbpp.uniceub.br

Observe as normas de publicação, para facilitar e agilizar o trabalho de edição. 